\title{
Energy Transfer and Emission Decay Kinetics in Mixed Microporous Lanthanide Silicates with Unusual Dimensionality
}

\author{
Rachel C. Evans, $*,+,, \S$ Duarte Ananias, ${ }^{\ddagger, l}$ Alastair Douglas, ${ }^{\perp}$ Peter Douglas, ${ }^{\dagger}$ \\ Luis D. Carlos, *, and João Rocha \\ Department of Chemistry, University of Wales Swansea, Singleton Park, Swansea, SA2 8PP, UK, Department \\ of Chemistry and Department of Physics, University of Aveiro, CICECO, 3810-193 Aveiro, Portugal, \\ Department of Biochemistry, NMR Centre and Centre of Neurosciences and Cell Biology, University of \\ Coimbra, 3001-401 Coimbra, Portugal, and AD Technology Consulting Limited, Swansea, SA2 8PP, UK
}

Received: June 18, 2007; In Final Form: October 3, 2007

\begin{abstract}
We have investigated the energy transfer dynamics in mixed lanthanide open-framework silicates, known as Ln-AV-20 materials, with the stoichiometric formula $\mathrm{Na}_{1.08} \mathrm{~K}_{0.5} \mathrm{Ln}_{1.14} \mathrm{Si}_{3} \mathrm{O}_{8.5}{ }^{\circ} 1.78 \mathrm{H}_{2} \mathrm{O}\left(\mathrm{Ln}=\mathrm{Gd}^{3+}, \mathrm{Tb}^{3+}, \mathrm{Eu}^{3+}\right)$, using steady-state and time-resolved luminescence spectroscopy. Energy transfer between donor and acceptor $\mathrm{Ln}^{3+}$ ions is extremely efficient, even at low molar ratios of the acceptor $\mathrm{Ln}^{3+}(<5 \%)$. The presence of two different $\mathrm{Ln}^{3+}$ environments makes the Ln-AV-20 intralayer structure intermediate between purely onedimensional (1D) and two-dimensional (2D). The unusual dimensionality of the Ln-AV-20 layers prevents modeling of energy transfer kinetics by conventional kinetic models. We have developed a computer modeling program for the analysis of energy transfer kinetics in systems of unusual dimensions and show how it may be applied successfully to the AV-20 system. Using the program, nearest neighbor energy transfer rate constants are calculated as $(5.30 \pm 0.07) \times 10^{6}$ and $(6.00 \pm 0.13) \times 10^{6} \mathrm{~s}^{-1}$, respectively, for $\mathrm{Gd} / \mathrm{Tb}-\mathrm{and} \mathrm{Tb} / \mathrm{Eu}-$ AV-20 at $300 \mathrm{~K}$. With increasing acceptor concentration, the energy transfer dynamics tend toward purely one-dimensional behavior, and thus, with careful selection of the ratio of individual $\mathrm{Ln}^{3+}$ ions, it is possible to tune the energy transfer dimensionality of the AV-20 layers from pure 1D to something intermediate between $1 \mathrm{D}$ and $2 \mathrm{D}$.
\end{abstract}

\section{Introduction}

Luminescent materials that emit the three primary colors (red, green, and blue) are required for flat panel display and lighting technology, where a high spatial resolution and wide color range is desirable. ${ }^{1,2}$ Classical luminescent solid-state materials are increasingly giving way to new materials, and some of our previous efforts in this context have focused on the chemistry of novel zeo-type materials, exhibiting a microporous framework but encompassing stoichiometric amounts of transition or lanthanide ions. ${ }^{3-8}$ These materials are interesting because, depending on the framework elements incorporated, they may combine, in a single stable solid, both microporosity and tunable optical properties. ${ }^{4-8}$

Lanthanide ( $\mathrm{Ln}$ )-containing materials are ideal for display technology, since, with the judicious selection of red- $\left(\mathrm{Eu}^{3+}\right.$, $\left.\mathrm{Pr}^{3+}, \mathrm{Sm}^{3+}\right)$, green- $\left(\mathrm{Tb}^{3+}, \mathrm{Er}^{3+}\right)$, and blue- $\left(\mathrm{Tm}^{3+}, \mathrm{Ce}^{3+}\right)$ emitting ions doped in an inert host, it is possible to design phosphors that emit across the entire visible spectrum with high color purity. 2,9 The incorporation of more than one type of $\mathrm{Ln}^{3+}$ ion into a host lattice introduces not only the possibility of obtaining luminescence in different parts of the visible spectrum but also the opportunity of the transfer of excitation energy

* Corresponding authors. R.C.E.: tel, +44-1792-513081; fax, +44-1792 295747; e-mail, rachelcevans@yahoo.co.uk. L.D.C.: tel, +351-234370946; fax, +351-234424965; e-mail, 1carlos@ fis.ua.pt.

University of Wales Swansea.

$\doteqdot$ Department of Chemistry, University of Aveiro.

$\S$ Department of Physics, University of Aveiro.

"University of Coimbra.

$\perp$ AD Technology Consulting Ltd. between the different $\mathrm{Ln}^{3+}$ ions, provided that there is a sufficient overlap of energy levels. ${ }^{10-14}$

Recently, some of us reported the synthesis, structure, and photoluminescence (PL) properties of some Ln silicates $\left(\mathrm{Na}_{1.08} \mathrm{~K}_{0.5} \mathrm{Ln}_{1.14} \mathrm{Si}_{3} \mathrm{O}_{8.5} \cdot 1.78 \mathrm{H}_{2} \mathrm{O}, \mathrm{Ln}=\mathrm{Gd}^{3+}, \mathrm{Eu}^{3+}, \mathrm{Tb}^{3+}, \mathrm{Sm}^{3+}\right.$, $\mathrm{Ce}^{3+}$ ), named Ln-AV-20 materials. ${ }^{5}$ The Ln-AV-20 structure is very versatile in that it allows the introduction of a mixture of lanthanide ions into the framework without changing the crystal phase. Furthermore, extremely efficient energy transfer is observed between different $\mathrm{Ln}^{3+}$ ion pairs in mixed $\mathrm{Ln}-\mathrm{AV}$ 20 systems (e.g. Tb/Eu or $\mathrm{Gd} / \mathrm{Tb}$ ). ${ }^{5}$ Although in principle energy transfer introduces the possibility of fine-tuning the photoluminescence properties of the system, to our knowledge there have been no comprehensive studies exploring the dynamics of energy transfer in either Ln-AV-20 or related stoichiometric lanthanide silicate systems.

Here, by studying both the steady-state and nanosecond timeresolved photoluminescence dynamics, we investigate the kinetics of energy transfer in $\mathrm{Gd}_{1-x} \mathrm{~Tb}_{x^{-}}$and $\mathrm{Tb}_{1-x} \mathrm{Eu}-\mathrm{AV}-20$, with the aim of obtaining information to help fine-tune the photoluminescence properties of these and related novel materials. Studying the energy transfer dynamics of such systems is not trivial. The presence of two Ln environments creates an unusual dimensionality within the AV-20 structure, which prevents the study of this system using conventional one-, two-, or threedimensional (1D, 2D, or 3D) energy transfer models. A computer modeling program (known herein as ProgClusters) for the analysis of energy transfer kinetics in systems of unusual dimensions is introduced. We show how ProgClusters may be successfully applied to model the energy transfer dynamics in 


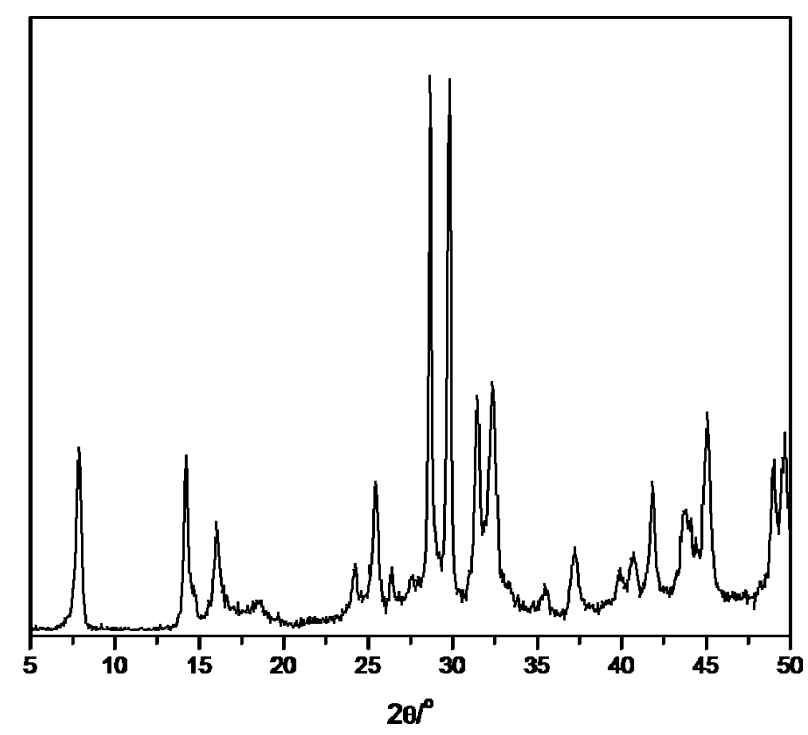

Figure 1. Powder XRD pattern of $\mathrm{Gd}_{1-x} \mathrm{~Tb}_{x}-\mathrm{AV}-20(x=0.20)$.

mixed Ln-AV-20 systems. The validity of this approach to model the Ln-AV-20 and related systems is discussed and its potential as a pre-experimentation design tool is considered.

\section{Experimental Methods}

2.1. Synthesis. The synthesis of AV-20 materials was performed using the method described previously. ${ }^{5}$ In a typical Eu-AV-20 $\left(\mathrm{Na}_{1.08} \mathrm{~K}_{0.5} \mathrm{Eu}_{1.14} \mathrm{Si}_{3} \mathrm{O}_{8.5} \cdot 1.78 \mathrm{H}_{2} \mathrm{O}\right)$ synthesis, an alkaline solution was made by mixing $5.75 \mathrm{~g}$ of sodium silicate solution $\left(27 \% \mathrm{~m} / \mathrm{m} \mathrm{SiO}_{2}, 8 \% \mathrm{~m} / \mathrm{m} \mathrm{Na}_{2} \mathrm{O}\right.$, Merck), $16.50 \mathrm{~g}$ of $\mathrm{H}_{2} \mathrm{O}, 3.25 \mathrm{~g}$ of $\mathrm{KOH}$ (Merck), and $1.07 \mathrm{~g}$ of $\mathrm{NaOH}$ (Merck); $1.37 \mathrm{~g}$ of $\mathrm{EuCl}_{3} \cdot 6 \mathrm{H}_{2} \mathrm{O}$ (Aldrich) was added to this solution and the mixture stirred thoroughly. The gel, with composition $0.79 \mathrm{Na}_{2} \mathrm{O}: 1.10 \mathrm{~K}_{2} \mathrm{O}: 1.0 \mathrm{SiO}_{2}: 0.07 \mathrm{Eu}_{2} \mathrm{O}_{3}: 35 \mathrm{H}_{2} \mathrm{O}$, was autoclaved under autogenous pressure for 3 days at $230{ }^{\circ} \mathrm{C}$. The off-white microcrystalline powders obtained were filtered, washed at room temperature with distilled water, and dried at $100{ }^{\circ} \mathrm{C}$. Tb- and Gd-AV-20 were synthesized with substitution of $\mathrm{EuCl}_{3} \cdot 6 \mathrm{H}_{2} \mathrm{O}$ by the corresponding $\mathrm{LnCl}_{3} \cdot 6 \mathrm{H}_{2} \mathrm{O}(\mathrm{Ln}=\mathrm{Gd}, \mathrm{Tb}$, Aldrich). Mixed $\mathrm{Gd}_{1-x} \mathrm{~Tb}_{x^{-}}(x=0.05,0.3)$ and $\mathrm{Tb}_{1-x} \mathrm{Eu}_{x}-\mathrm{AV}-20(x=$ $0.01,0.05,0.2,0.3,0.4,0.5)$ samples were prepared by introducing in the parent gel the desired molar ratio of the two lanthanides.

2.2. Characterization. Powder $X$-ray diffraction (XRD) data were collected on an X'Pert MPD Phillips diffractometer $(\mathrm{Cu}$ $\mathrm{K} \alpha$ radiation) fitted with a curved graphite monochromator, a fixed divergence slit of $1 / 8^{\circ}$, and a flat plate sample holder, in a Bragg-Brantano para-focusing optics configuration. Scanning electron microscopy (SEM) was performed using a Hitachi S-4100 microscope. Energy dispersive spectroscopy (EDS) was carried out using an EDS Rönteck System with polymeric window attached to the SEM.

Within experimental error, chemical analysis by EDS performed on six crystals for each sample confirmed the Ln:K: $\mathrm{Na}: \mathrm{Si}$ ratios (ca. 1:0.44:0.93:2.63) obtained previously by powder XRD. ${ }^{5}$ For mixed lanthanide samples, the relative $\mathrm{Ln}^{3+}$ ratio was consistent with the molar ratio introduced into the parent synthesis gel within experimental error. No crystals rich in an individual lanthanide were found. All results indicated that lanthanides are randomly distributed within the crystals. All materials studied display powder XRD patterns characteristic of AV-20 (Figure 1), and no evidence was found for the presence of any impurity phases. No significant changes in the unit cell parameters or crystal morphology were observed on incorporation of more than one type of lanthanide in the sample.

For photoluminescence measurements, Ln-AV-20 samples were ground into fine powders and then pressed under a pressure of 10 tonnes per $\mathrm{cm}^{2}$ into thin $(<1 \mathrm{~mm})$ circular discs with a diameter of $15 \mathrm{~mm}$. Steady-state photoluminescence measurements were performed on a Fluorolog-3 (FL3-2T model) with double excitation spectrometer, fitted with a 1200 groves $/ \mathrm{mm}$ grating blazed at $330 \mathrm{~nm}$, and a single emission spectrometer (TRIAX 320), fitted with a 1200 groves/mm grating blazed at $500 \mathrm{~nm}$, coupled to a R928P Hamamatsu photomultiplier. The excitation source was a $450 \mathrm{~W}$ Xe arc lamp. All emission spectra were corrected for the spectral response of the monochromators and the detector using typical correction spectra provided by the manufacturer. The temperature was controlled $(12-300 \mathrm{~K})$ using a He closed-cycle cryostat.

Time-resolved (ns) emission decay measurements were performed using a Nd/YAG Spectron laser with an Applied Photophysics laser kinetic spectrometer. Samples were excited at $355 \mathrm{~nm}\left(\mathrm{~Tb}_{1-x} \mathrm{Eu}_{x}-\mathrm{AV}-20\right)$ and $266 \mathrm{~nm}\left(\mathrm{Gd}_{1-x} \mathrm{~Tb}_{x}-\mathrm{AV}-20\right)$ using the third and fourth harmonics of the Q-switched laser, respectively. The average trace of 16 scans was collected on a 12 bit Picoscope analog to digital converter (Pico Technology Ltd., Cambridgeshire, UK), before transferring to a PC for analysis. The emission monochromator slits were set at $4 \mathrm{~mm}$ (corresponding to a bandwidth of $18.6 \mathrm{~nm}$ ).

2.3. Kinetic Modeling. The energy transfer dynamics in mixed Ln-AV-20 systems were investigated using a computer modeling program known as ProgClusters. ${ }^{15}$ Although the program was designed with the AV-20 system in mind, it is not specific to this structure and in principle may be applied to any system whose dimensionality ranges between $1 \mathrm{D}$ and $2 \mathrm{D}$. The program enables the user to set up a large array of donor (D) and acceptor (A) sites. The dimensionality of the array may be specified to represent that of the sample under study. ProgClusters models the energy transfer by a series of steps in which a small fraction of energy is transferred between nearestneighbor lanthanide ions. Transfer of energy from an excited donor to an energy acceptor/trap results in a corresponding decrease in the total donor excited-state population. The program allows the user to vary a number of empirical and modeling parameters.

We have used experimentally accessible values wherever possible for fitting parameters. Where this was not possible, best-fit parameters were obtained by exploration of values in small increments within a sensible range.

\section{Results and Discussion}

3.1. Structural Data. To assist with the discussion of the photoluminescence results, the Ln-AV-20 structure is now briefly described (Figure 2). A monoclinic unit cell ( $a=6.851$ $\AA, b=22.248 \AA, c=6.828 \AA, \beta=113.92^{\circ}$ ) and a $B 11 m$ space group have been previously reported for Eu-AV-20.5 The structure consists of a series of layers, in which each layer is built up of alternating chains of seven-coordinated (monocapped trigonal prisms) regular $\mathrm{Eu}^{3+}(\mathrm{Ln} 1)$ and $\mathrm{Na}^{+}$polyhedra running along the [110] direction. In addition, some (ca. 10\%) $\mathrm{Eu}^{3+}$ ions (Ln2) are disordered over the $\mathrm{Na}^{+}$sites. Neighboring chains in each layer present the capping ligands (oxygen and water molecules) on their opposite surfaces. In mixed lanthanide samples, the chains exhibit enough structural versatility to host at least two different ions, such as $\mathrm{Eu}^{3+}$ and $\mathrm{Tb}^{3+}$, or $\mathrm{Gd}^{3+}$ and $\mathrm{Tb}^{3+}$, without markedly changing the unit cell parameters. ${ }^{5}$

$\mathrm{Eu} / \mathrm{Na}$ polyhedra layers are connected through double silicate chains, which also run along the [110] direction, on both sides 

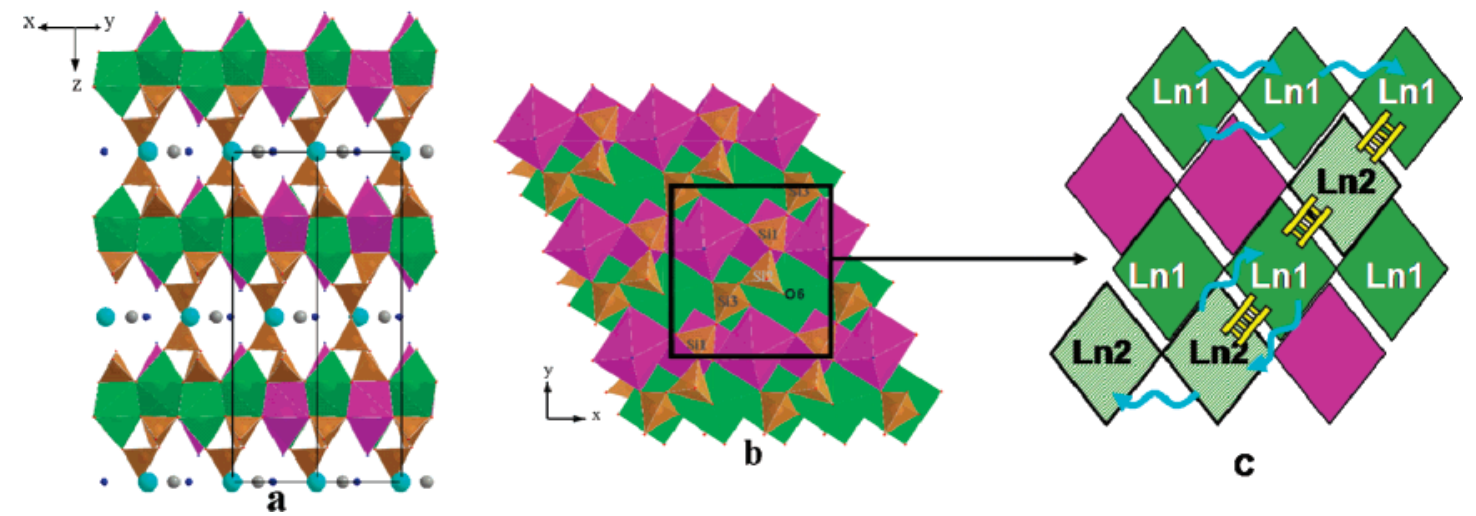

Figure 2. (a) Crystal structure of Eu-AV-20. (b) Connection of silicate chains to the layer of $\mathrm{EuO}_{7}$ and $\mathrm{NaO}_{7}$ polyhedra. Polyhedra: green (Ln1$\left.\mathrm{EuO}_{7}\right)$, green hatched $\left(\mathrm{Ln} 2-\mathrm{EuO}_{7}\right)$, purple $\left(\mathrm{NaO}_{6}\left(\mathrm{H}_{2} \mathrm{O}\right)\right)$, brown $\left(\mathrm{SiO}_{4}\right)$. Spheres: light blue $\left(\mathrm{KO}_{4}\left(\mathrm{H}_{2} \mathrm{O}\right)_{3}\right)$, light gray $\left(\mathrm{NaO}_{3}\left(\mathrm{H}_{2} \mathrm{O}\right)_{3}\right)$, blue $\left(\mathrm{H}_{2} \mathrm{O}\right)$. $(\mathrm{c})$ Cartoon representation of an AV-20 layer. The blue curly arrows indicate the possible energy transfer pathways: Ln1 $\rightarrow \operatorname{Ln} 1, \mathrm{Ln} 1 \rightarrow \operatorname{Ln} 2, \mathrm{Ln} 2 \rightarrow$ $\mathrm{Ln} 1$, and $\mathrm{Ln} 2 \rightarrow \mathrm{Ln} 2$. The yellow bridges illustrate the role of the Ln2 sites as interconnecting bridges through which energy may migrate between the otherwise isolated Ln1 chains.

of the $\mathrm{Eu}^{3+} / \mathrm{Na}^{+}$layers shifted by $(a+b) / 4$, thus building up a framework with channels running along the [110] direction. The siliceous chains are comprised of $\mathrm{Si}_{2} \mathrm{O}_{7}$ units linked through a bridging tetrahedron. Two of these chains are condensed through the symmetry plane normal to $c$ via a bridging oxygen to form the double silicate chain, resulting in the formation of an eightmembered ring. Each siliceous chain connects to a $\mathrm{Eu} / \mathrm{Na}$ chain by $\mathrm{Si}_{2} \mathrm{O}_{7}$ units sharing the oxygen atoms of the edges opposite to the capping ligands. The cavities of Eu-AV-20 contain $\mathrm{Na}^{+}$ and $\mathrm{K}^{+}$cations. $\mathrm{Na}^{+}$cations are six-coordinated to three framework oxygen atoms and three water molecules. Both sites may be partially occupied by water molecules.

It is important to note that due to the presence of two Ln environments, namely, the regular Ln polyhedra (Ln1) and the Ln polyhedra disordered over $\mathrm{Na}^{+}$sites (Ln2), the individual AV-20 layers are not simple one- or two-dimensional planar structures. In the absence of the Ln2 disordered sites, the AV20 layers would consist of a series of discrete one-dimensional chains of Ln1 polyhedra. The Ln2 sites act as bridges between the Ln1 chains, lifting the intralayer Ln environment from a one-dimensional chain to an intermediate one-two-dimensional regime.

3.2. Steady-State Photoluminescence. Ln-AV-20 systems $\left(\mathrm{Ln}=\mathrm{Gd}^{3+}, \mathrm{Tb}^{3+}, \mathrm{Eu}^{3+}\right)$ where all available $\mathrm{Ln}^{3+}$ sites, both regular and disordered, are occupied by a single type of $\mathrm{Ln}^{3+}$ exhibit photoluminescence spectra characteristic of the ion present. ${ }^{5}$ The photoluminescence spectra of the mixed lanthanides $\mathrm{Gd}_{0.95} \mathrm{~Tb}_{0.05}-\mathrm{AV}-20$ and $\mathrm{Tb}_{0.95} \mathrm{Eu}_{0.05}-\mathrm{AV}-20$ selectively excited respectively in the $\mathrm{Gd}^{3+}(313 \mathrm{~nm})$ and the $\mathrm{Tb}^{3+}(351$ $\mathrm{nm}$ ) levels are shown in Figure 3. The $\mathrm{Gd}_{0.95} \mathrm{~Tb}_{0.05} \mathrm{AV}-20$ spectrum exhibits the characteristic $\mathrm{Tb}^{3+}{ }^{5} \mathrm{D}_{4} \rightarrow{ }^{7} \mathrm{~F}_{6-2}$ transitions observed for Tb-AV-20.5 Similarly, the $\mathrm{Tb}_{0.95} \mathrm{Eu}_{0.05} \mathrm{AV}-20$ spectrum exhibits the characteristic $\mathrm{Eu}^{3+}{ }^{5} \mathrm{D}_{0} \rightarrow{ }^{7} \mathrm{~F}_{0-4}$ transition lines. The occurrence of this mode of energy transfer indicates that the two types of lanthanide optical centers are close and that there is no separation of lanthanides into separate crystal domains. These observations concur with previously reported energy transfer in the related $\mathrm{Gd}_{0.5} \mathrm{~Tb}_{0.5^{-}}$and $\mathrm{Tb}_{0.5} \mathrm{Eu}_{0.5^{-}} \mathrm{AV}-20$ systems. ${ }^{5}$ What is interesting to note is that, with just $5 \%$ doping of the second $\mathrm{Ln}^{3+}$ acceptor ion $\left(\mathrm{Tb}^{3+}, \mathrm{Eu}^{3+}\right)$, emission from the major $\mathrm{Ln}^{3+}$ ion is drastically quenched, indicating extremely efficient energy transfer between ions. Indeed, on increasing the substitution of the acceptor ion to $20 \%$, emission of the donor $\mathrm{Ln}^{3+}$ ion is undetectable with our experimental arrangement. The inset of Figure 3 shows the $\mathrm{Eu}^{3+}{ }^{5} \mathrm{D}_{0} \rightarrow{ }^{7} \mathrm{~F}_{0}$ transition observed for $\mathrm{Tb}_{0.95} \mathrm{Eu}_{0.05}-\mathrm{AV}-20$. The $\mathrm{Eu}^{3+}$ ion is unique in that

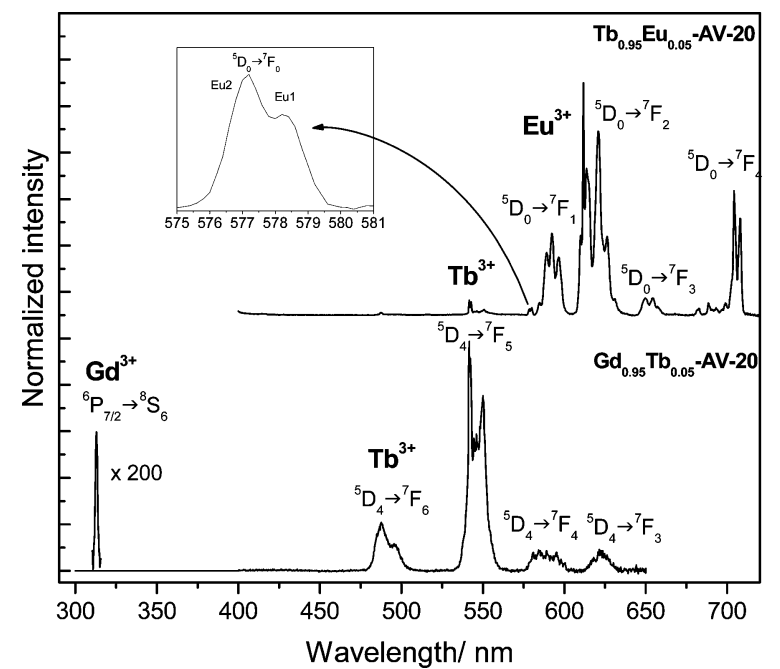

Figure 3. $300 \mathrm{~K}$ emission spectra of $\mathrm{Tb}_{0.95} \mathrm{Eu}_{0.05}-\mathrm{AV}-20$ and $\mathrm{Gd}_{0.95}$ $\mathrm{Tb}_{0.05}$-AV-20 excited at $351 \mathrm{~nm}\left({ }^{5} \mathrm{D}_{2}, \mathrm{~Tb}^{3+}\right)$ and $273 \mathrm{~nm}\left({ }^{6} \mathrm{I}_{9 / 2}, \mathrm{Gd}^{3+}\right)$ respectively. The inset shows a vertical expansion of the $\mathrm{Eu}^{3+5} \mathrm{D}_{0} \rightarrow$ ${ }^{7} \mathrm{~F}_{0}$ transition observed for $\mathrm{Tb}_{0.95} \mathrm{Eu}_{0.05}-\mathrm{AV}-20$.

both the ground state $\left({ }^{7} \mathrm{~F}_{0}\right)$ and the excited emissive state $\left({ }^{5} \mathrm{D}_{0}\right)$ are nondegenerate and neither state may be split by the crystal field. This implies that there is a one-to-one correspondence between the number of peaks associated with the ${ }^{7} \mathrm{~F}_{0} \rightarrow{ }^{5} \mathrm{D}_{0}$ transition in the emission spectrum and the number of distinct $\mathrm{Eu}^{3+}$ environments. ${ }^{16}$ Gaussian deconvolution of the ${ }^{5} \mathrm{D}_{0} \rightarrow{ }^{7} \mathrm{~F}_{0}$ transition suggests that two local $\mathrm{Eu}^{3+}$ environments are present, with maxima at 577.2 and $578.2 \mathrm{~nm}$, respectively, which is in accordance with the data reported for Eu-AV-20.5 Furthermore, the presence of two $\mathrm{Eu}^{3+}$ local environments, even at a low $\mathrm{Eu}^{3+}$ concentration, indicates that there is no selectivity for a specific $\mathrm{Ln}^{3+}$ ion to occupy a particular site.

3.3. Emission Decay Dynamics. Figure 4 shows the timeresolved emission decay curves obtained for $\mathrm{Gd}_{1-x} \mathrm{~Tb}_{x}-\mathrm{AV}-20$ $(x=0.05,0.30)$ following excitation into the ${ }^{6} \mathrm{I}_{11 / 2} \mathrm{Gd}^{3+}$ level $(266 \mathrm{~nm})$ at $300 \mathrm{~K}$. Dynamic energy transfer from $\mathrm{Gd}^{3+}$ toTb ${ }^{3+}$ is most convincingly shown by the excitation grow-in on the decay curves detected at the $\mathrm{Tb}^{3+}{ }^{5} \mathrm{D}_{4}$ state for $x=0.05$. The observed decay rate of the corresponding $\mathrm{Gd}^{3+}{ }^{6} \mathrm{P}_{7 / 2}$ emission for this sample is of the same order of magnitude as the rise time of the $\mathrm{Tb}^{3+}\left({ }^{5} \mathrm{D}_{4}\right)$ emission. On increasing the $\mathrm{Tb}^{3+}$ concentration, the $\mathrm{Gd}^{3+}{ }^{6} \mathrm{P}_{7 / 2}$ emission becomes increasingly quenched, such that at concentrations above $x=0.3$ the $\mathrm{Gd}^{3+}$ luminescence was too weak for measurement in our experi- 


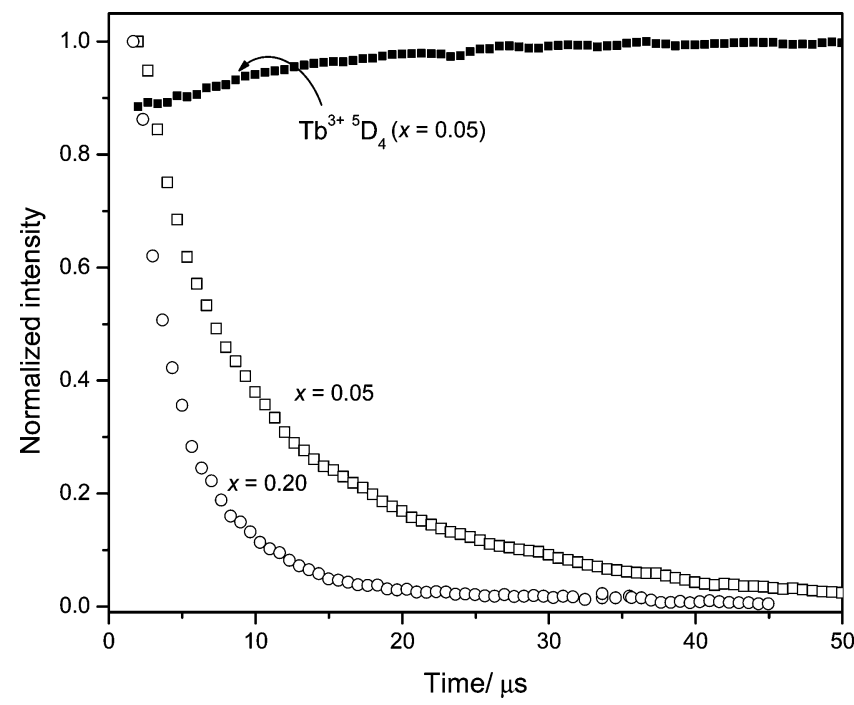

Figure 4. Normalized $300 \mathrm{~K}$ emission decay curves for $\mathrm{Gd}_{1-x} \mathrm{~Tb}_{x^{-}}$ AV-20 excited at $266 \mathrm{~nm}$ and detected at $313 \mathrm{~nm}\left(\mathrm{Gd}^{3+},{ }^{6} \mathrm{P}_{7 / 2} \rightarrow{ }^{8} \mathrm{~S}\right)$ for $x=0.05$ (squares) and $x=0.3$ (circles). The filled squares represent the grow-in of the $\mathrm{Tb}^{3+}$ emission detected at $542 \mathrm{~nm}\left({ }^{5} \mathrm{D}_{4} \rightarrow{ }^{7} \mathrm{~F}_{5}\right)$ for $x=0.05$.

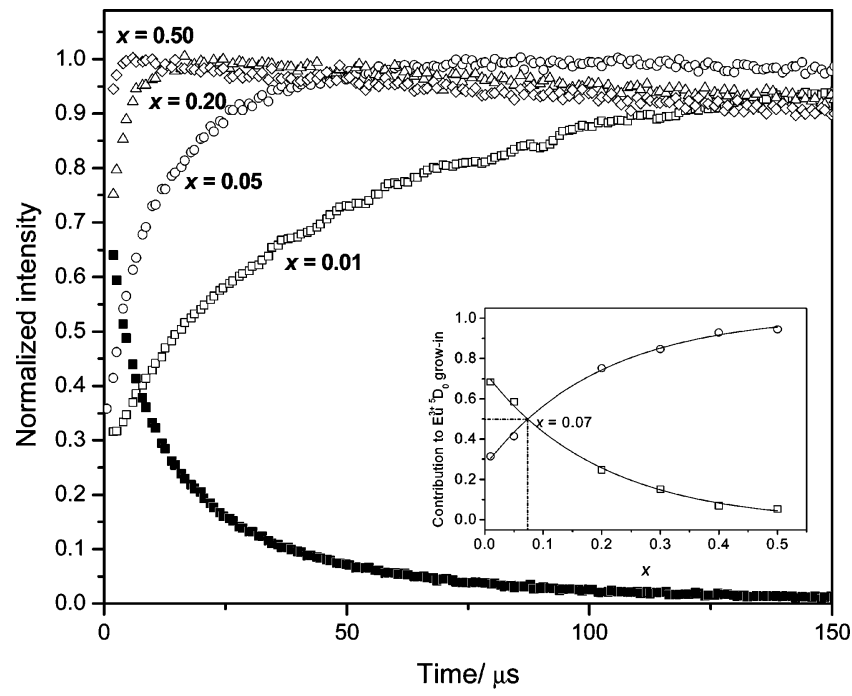

Figure 5. Emission intensity grow-in curves for $\mathrm{Tb}_{1-x} \mathrm{Eu}_{x}-\mathrm{AV}-20$ samples excited at $355 \mathrm{~nm}\left(\mathrm{~Tb}^{3+},{ }^{5} \mathrm{D}_{2}\right)$ and detected at $704 \mathrm{~nm}\left(\mathrm{Eu}^{3+}\right.$, ${ }^{5} \mathrm{D}_{0} \rightarrow{ }^{7} \mathrm{~F}_{4}$ ). The corresponding $\mathrm{Tb}^{3+}{ }^{5} \mathrm{D}_{4}$ decay curve (filled squares) for $x=0.05$ is included for comparison. The inset shows the fractional contribution (determined from the ratio of emission intensities at $t=$ $1.92 \mu \mathrm{s}$ and at the end of the emission grow-in) of (i) static shortrange $\mathrm{Tb}^{3+} \rightarrow \mathrm{Eu}^{3+}$ energy transfer (circles) and (ii) dynamic $\mathrm{Tb}^{3+} \rightarrow$ $\mathrm{Eu}^{3+}$ energy transfer (squares) to the $\mathrm{Eu}^{3+5} \mathrm{D}_{0}$ level excitation intensity grow-in as a function of $\mathrm{Eu}^{3+}$ concentration $(x)$. The solid lines serve only to guide the eye.

mental setup. The absence of an emission rise time in the $\mathrm{Tb}^{3+}$ decay curves for samples containing $\mathrm{Tb}^{3+}$ concentrations greater than $x=0.05$ indicates that $\mathrm{Gd}^{3+} \rightarrow \mathrm{Tb}^{3+}$ energy transfer occurs on a time scale beyond the limit of detection of our instrumentation (ca. $<100 \mathrm{~ns}$ ). Figure 5 shows the normalized emission grow-in traces for the $\mathrm{Eu}^{3+5} \mathrm{D}_{0}$ emission obtained on excitation into the $\mathrm{Tb}^{3+}\left({ }^{5} \mathrm{D}_{2}\right)$ level $(355 \mathrm{~nm})$ for $\mathrm{Tb}_{1-x} \mathrm{Eu}_{x}-\mathrm{AV}-20(x=$ 0.01-0.5) samples. To enable comparison, the decay curves are shown until $150 \mu$ s after the excitation pulse. With increasing $\mathrm{Eu}^{3+}$ concentration a gradual decrease in the emission rise time from ca. $300 \mu$ s for $x=0.01$ to ca. $5 \mu$ s for $x=0.5$ is observed, indicating that the rate of $\mathrm{Tb}^{3+} \rightarrow \mathrm{Eu}^{3+}$ energy transfer is dependent on the $\mathrm{Eu}^{3+}$ concentration. For all samples, the rise time of the $\mathrm{Eu}^{3+}$ emission is comparable to the corresponding observed emission decay time of the $\mathrm{Tb}^{3+}{ }^{5} \mathrm{D}_{4}$ level (shown in Figure 5 for $x=0.01$ ).

The absolute intensity of the $\mathrm{Eu}^{3+}{ }^{5} \mathrm{D}_{0}$ grow-in curves is not zero at the time of the first data point we can collect with this experimental arrangement $(t=2.0 \mu \mathrm{s})$. Excitation at $355 \mathrm{~nm}$ enables population of the $\mathrm{Eu}^{3+}{ }^{5} \mathrm{D}_{0}$ level via a combination of (i) $\mathrm{Tb}^{3+} \rightarrow \mathrm{Eu}^{3+}$ energy transfer occurring faster than the time resolution of this experiment (i.e., between $t=0$ and $2.0 \mu \mathrm{s}$ ), which we will refer to as static energy, since it involves short distance energy transfer between adjacent and close neighbor donor-acceptor pairs and for this fast energy transfer our experiments give no time-resolved information, and (ii) dynamic $\mathrm{Tb}^{3+} \rightarrow \mathrm{Eu}^{3+}$ energy transfer following migration among $\mathrm{Tb}^{3+}$ donors occurring after $t=2.0 \mu \mathrm{s}$. The inset of Figure 5 shows a plot of the fractional contribution from (i) direct excitation/ static $\mathrm{Tb}^{3+} \rightarrow \mathrm{Eu}^{3+}$ energy transfer and (ii) dynamic $\mathrm{Tb}^{3+} \rightarrow$ $\mathrm{Eu}^{3+}$ energy transfer to the excitation intensity grow-in as a function of $\mathrm{Eu}^{3+}$ concentration. As the $\mathrm{Eu}^{3+}$ concentration is increased, a corresponding rise in the fraction of $\mathrm{Eu}^{3+}$ sites populated by direct excitation or static $\mathrm{Tb}^{3+} \rightarrow \mathrm{Eu}^{3+}$ energy transfer is observed. This is accompanied by a parallel decrease in the fraction of $\mathrm{Eu}^{3+}$ sites populated by dynamic $\mathrm{Tb}^{3+} \rightarrow \mathrm{Eu}^{3+}$ energy transfer, as a consequence of diffusion of excitation energy across the $\mathrm{Tb}^{3+}$ donors. At $x \approx 0.07$, the fraction of $\mathrm{Eu}^{3+}$ sites populated via direct/static and dynamic energy transfer processes is equal. The concentration dependence of the $\mathrm{Eu}^{3+}$ population mechanism is sensible; at lower concentrations, there are fewer nearest neighbor $\mathrm{Tb}^{3+}-\mathrm{Eu}^{3+}$ pairs and energy must first migrate over the $\mathrm{Tb}^{3+}$ donor system before reaching an acceptor. As the $\mathrm{Eu}^{3+}$ concentration increases, the number of $\mathrm{Tb}^{3+}-\mathrm{Eu}^{3+}$ nearest neighbors will also increase, as will the probability of static energy transfer.

Figure 6a shows the normalized emission decay curves (300 $\mathrm{K})$ obtained for $\mathrm{Tb}_{1-x} \mathrm{Eu}_{x}-\mathrm{AV}-20$ systems excited and detected in the $\mathrm{Tb}^{3+}{ }^{5} \mathrm{D}_{4}$ level. On increasing the $\mathrm{Eu}^{3+}$ concentration from $x=0.01$ to 0.5 , significant quenching of the lifetime of the $\mathrm{Tb}^{3+5} \mathrm{D}_{4}$ state is observed, indicative of $\mathrm{Tb}^{3+} \rightarrow \mathrm{Eu}^{3+}$ energy transfer. The decay traces can be resolved into an initial fast decay and a longer-lived slow decay component. This kinetic form is characteristic of diffusion-limited energy transfer. ${ }^{17}$ The Ln-AV-20 structure may be considered as a system of energy donors containing a uniform, random distribution of energy acceptor sites. In this condition, energy migration among donors occurs at a rate comparable to the intrinsic decay rate of the excited state. Excited donors with acceptors as nearest neighbors relax predominantly via direct energy transfer, while isolated donors must first transfer their excitation energy through the donor system before $\mathrm{D} \rightarrow$ A energy transfer may occur. On short time scales, the contribution of energy migration among donors to the decay kinetics is negligible and the luminescence decay curve exhibits a fast nonexponential component characteristic of direct energy transfer. At longer decay times, energy migration becomes more important and the decay kinetics approaches monoexponential behavior. ${ }^{17}$

Figure $6 \mathrm{~b}$ shows the corresponding emission decay curves obtained for detection in the $\mathrm{Eu}^{3+5} \mathrm{D}_{0} \rightarrow{ }^{7} \mathrm{~F}_{4}$ transition. To aid comparison, the emission decays are normalized to approximately the start of the emission decay by subtraction of the corresponding rise time observed for that sample. A gradual decrease in the lifetime of the $\mathrm{Eu}^{3+}{ }^{5} \mathrm{D}_{0}$ emissive level is observed with increasing $\mathrm{Eu}^{3+}$ concentration. Concentration quenching, where excitation energy migrates between $\mathrm{Ln}^{3+}$ sites before being trapped by an energy quencher, is a common phenomenon in systems where the lanthanide concentration is 


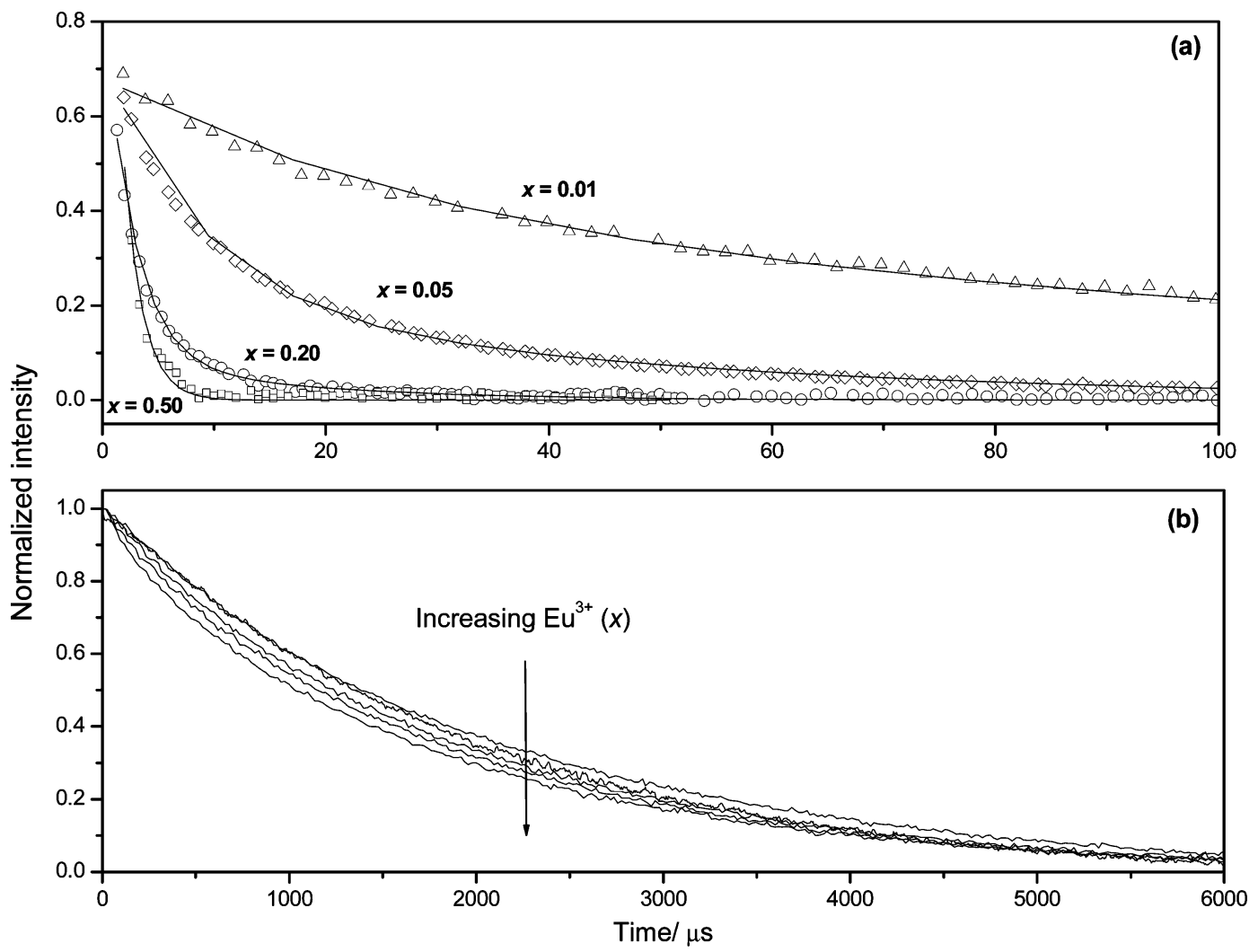

Figure 6. Normalized emission decay curves for $\mathrm{Tb}_{1-x} \mathrm{Eu}_{x}-\mathrm{AV}-20$ excited at $355 \mathrm{~nm}$; (a) $x=0.01,0.05,0.20$, and 0.50 , detected at $542 \mathrm{~nm}$ ( $\mathrm{Tb}^{3+}$, ${ }^{5} \mathrm{D}_{4} \rightarrow{ }^{7} \mathrm{~F}_{5}$ ). The curves are normalized to $t=0$ by correcting for the initial intensity at the start of the Eu ${ }^{3+}$ excitation grow-in. The solid lines serve only to guide the eye. (b) $x=0.01,0.05,0.20,0.40,0.50$, detected at $704 \mathrm{~nm}\left(\mathrm{Eu}^{3+},{ }^{5} \mathrm{D}_{0} \rightarrow{ }^{7} \mathrm{~F}_{4}\right)$. Curves are normalized to the start of the emission decay by subtraction of the rise time.

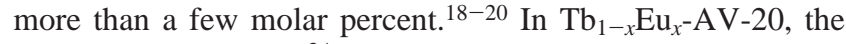
distribution of the $\mathrm{Eu}^{3+}$ acceptor ions that can be excited by $\mathrm{Tb}^{3+} \rightarrow \mathrm{Eu}^{3+}$ energy transfer is not random. The percolation model is a useful approach for describing energy migration processes in lanthanide systems. ${ }^{19,20}$ In this model it is assumed that the between $\mathrm{Ln}^{3+}$ ions occur only among nearest-neighbor sites in the sublattice. At low acceptor concentrations, it is assumed that the $\mathrm{Eu}^{3+}$ ions exist as individual ions or in small clusters within the $\mathrm{Tb}^{3+}$ donor system and $\mathrm{D} \rightarrow \mathrm{A}$ energy transfer occurs between these isolated acceptor ions and nearestneighbor donors. As the $\mathrm{Eu}^{3+}$ concentration increases, the size of the acceptor clusters within the donor system also increases. Only acceptor ions on the edge of the cluster can be directly populated by $\mathrm{Tb}^{3+} \rightarrow \mathrm{Eu}^{3+}$ energy transfer; however, acceptor ions within the center of the cluster may then be excited by $\mathrm{Eu}^{3+} \rightarrow \mathrm{Eu}^{3+}$ energy migration. Above a critical acceptor concentration, the acceptor clusters are all connected and the excitation energy is free to explore the entire lattice, increasing the probability of migration to energy traps.

Assuming first-order processes, the average D $\rightarrow$ A energy transfer rate constant $\left(k_{\mathrm{DA}}\right)$ for our dynamic energy transfer may be estimated from ${ }^{11}$

$$
k_{\mathrm{DA}}=k_{\mathrm{obs}}-k_{0}
$$

where $k_{0}$ is the donor intrinsic decay rate constant in the absence of energy transfer processes and $k_{\mathrm{obs}}$ is the observed donor decay rate constant where an energy acceptor is present with concentration $x . k_{\mathrm{obs}}$ was determined for $\mathrm{Tb}_{1-x} \mathrm{Eu}_{x}-\mathrm{AV}-20$ from a single exponential analysis of the tail of the donor decay curves which approach first-order kinetics, and $k_{0}$ was taken as $(2.50 \pm 0.02)$ $\times 10^{2} \mathrm{~s}^{-1}$, the emission decay rate constant determined for $\mathrm{Tb}^{3+}$ $\left({ }^{5} \mathrm{D}_{4}\right)$ in the absence of concentration quenching for Tb-AV-
$20 .^{21}$ The calculated $\mathrm{D} \rightarrow$ A energy transfer rate constants for $\mathrm{Tb}_{1-x} \mathrm{Eu}_{x}-\mathrm{AV}-20$ are shown in Table $1 . k_{\mathrm{DA}}$ increases proportionally with increasing acceptor concentration, which is attributed to the corresponding decreasing average donoracceptor distance.

3.4. Modeling the Energy Transfer Kinetics. The donor ion decay curves observed for mixed Ln-AV-20 systems (Ln $\left.=\mathrm{Gd}^{3+} / \mathrm{Tb}^{3+}, \mathrm{Tb}^{3+} / \mathrm{Eu}^{3+}\right)$ are characteristic of diffusion-limited $\mathrm{D} \rightarrow$ A energy transfer. ${ }^{17}$ In this condition, the rate of energy migration within the donor system is slower than the $\mathrm{D} \rightarrow \mathrm{A}$ energy transfer rate but still comparable to the donor intrinsic decay rate. The rate-determining step for $\mathrm{D} \rightarrow$ A energy transfer will be the diffusion of energy among the donor system. The nearest Ln1-Ln1 distance within the AV-20 layers is 3.951 $\AA .{ }^{5}$ The average distance between $\mathrm{Ln}^{3+}$ sites in different layers is ca. $10 \AA^{5}$ Assuming that $\mathrm{D} \rightarrow \mathrm{D}$ energy migration proceeds via a dipole-dipole interaction $\left(R^{-6}\right.$ dependence $),{ }^{23}$ the intralayer transfer probability compared to the interlayer transfer probability is on the order of 260:1. This implies that the diffusion of excitation energy will be far from isotropic across the three-dimensional crystal, and in Ln-AV-20 systems, it is extremely likely that intralayer energy transfer will dominate the decay kinetics. This form of quasi-two-dimensional energy transfer has been described for energy migration in EuTa $\mathrm{O}_{19}$ and $\mathrm{EuOCl}$, where the intralayer to interlayer transfer probability ratios are 24.4:1 and 6.9:1, respectively. ${ }^{24,25}$

Neglecting back-transfer from an acceptor, the two-dimensional (2D) diffusion model to describe the emission intensity at time, $t$, after the excitation pulse is given by ${ }^{26}$

$$
I(t)=I_{0} \exp \frac{\left(-k_{\mathrm{r}} t\right)}{\left(4 \pi C_{\mathrm{a}} a^{-2} D t\right)}
$$


TABLE 1: Summary of Fitting Parameters for the 300 K Donor Ion Photoluminescence Decay Curves for $\mathrm{Tb}_{1-x} \mathrm{Eu}_{x}-\mathrm{AV}-20$ and $\mathbf{G d}_{1-x} \mathbf{T b}_{x}-\mathrm{AV}-20$

\begin{tabular}{|c|c|c|c|c|c|c|c|c|}
\hline system & $x$ & $k_{\mathrm{obs}} / 10^{4} \mathrm{~s}^{-1 a}$ & $k_{\mathrm{DA}} / 10^{4} \mathrm{~s}^{-1 b}$ & $I_{\mathrm{F}}^{c}$ & $t_{\mathrm{h}} / 10^{-} 9 \mathrm{~s}^{c}$ & $k_{\text {nat }} / \mathrm{s}^{-1 c}$ & $k_{\mathrm{NN}} / 10^{6} \mathrm{~s}^{-1 d}$ & $n_{\text {hop }}^{e}$ \\
\hline \multirow[t]{5}{*}{$\mathrm{Tb}_{1-x} \mathrm{Eu}_{x}-\mathrm{AV}-20$} & 0.01 & $0.60 \pm 0.01$ & $0.58 \pm 0.01$ & 0.98 & $3.71 \pm 0.21$ & 50 & $6.84 \pm 0.40$ & 1043 \\
\hline & 0.05 & $2.18 \pm 0.02$ & $2.15 \pm 0.01$ & 0.71 & $4.95 \pm 0.17$ & 50 & $5.21 \pm 0.10$ & 273 \\
\hline & 0.20 & $7.89 \pm 0.52$ & $7.87 \pm 0.01$ & 0.39 & $4.75 \pm 0.13$ & 50 & $5.33 \pm 0.14$ & 68 \\
\hline & 0.30 & $17.51 \pm 1.19$ & $17.49 \pm 0.01$ & 0.16 & $4.73 \pm 0.21$ & 50 & $5.36 \pm 0.24$ & 31 \\
\hline & 0.40 & $34.72 \pm 1.99$ & $34.7 \pm 0.01$ & 0.09 & $4.80 \pm 0.12$ & 50 & $5.28 \pm 0.11$ & 15 \\
\hline \multirow[t]{2}{*}{$\mathrm{Gd}_{1-x} \mathrm{~Tb}_{x}-\mathrm{AV}-20$} & 0.05 & & & 0.60 & $4.19 \pm 0.21$ & 800 & $6.05 \pm 0.31$ & \\
\hline & 0.30 & & & 0.10 & $4.30 \pm 0.10$ & 800 & $5.86 \pm 0.17$ & \\
\hline
\end{tabular}

${ }^{a} k_{\mathrm{obs}}$ is the observed rate constant of the donor ion obtained from a single-exponential fit to the tail of the decay curve ( $>2 \mathrm{~ms}$ ). ${ }^{b} k_{\mathrm{DA}}$ is the average $\mathrm{D} \rightarrow$ A energy transfer rate constant for dynamic energy transfer calculated from eq $1 .{ }^{c} \mathrm{I}_{\mathrm{F}}, t_{\mathrm{h}}$, and $k_{\text {nat }}$ are the fraction of excited donor states remaining after the dead time of the experiment, the step time, and the natural donor decay rate constant, respectively (ProgClusters fitting parameters). ${ }^{d} k_{\mathrm{NN}}$ is the nearest-neighbor energy transfer rate constant calculated from eq $6 .{ }^{e} n_{\text {hop }}$ is the approximate number of energy hops for dynamic energy transfer before energy transfer to an acceptor calculated from eq 7.

where $I_{0}$ is the emission intensity at $t=0, k_{\mathrm{r}}$ is the radiative decay rate, $a$ is the $\mathrm{Ln}^{3+}$ interatomic distance, and $D$ is the diffusion constant. This model is only valid for low acceptor concentrations and assumes that donor and acceptor ions are both randomly distributed in the lattice, such that each site has a probability of being occupied by an acceptor $\left(C_{\mathrm{a}}\right)$ or a donor $\left(C_{\mathrm{d}}\right)$ ion given by $C_{\mathrm{a}}+C_{\mathrm{d}} \leq 1$. However, the $2 \mathrm{D}$ diffusion model completely fails to reproduce the shape of the $\mathrm{Tb}^{3+}$ emission decay curve for $\mathrm{Tb}_{0.99} \mathrm{Eu}_{0.01}-\mathrm{AV}-20$. This is not unexpected, as the Ln-AV-20 layers are not strictly twodimensional. The presence of Ln2 bridging sites lifts the dimensionality of the Ln-AV-20 layer from a purely 1D chain system to an intermediate $1 \mathrm{D}-2 \mathrm{D}$ regime. However, since the relative number of $\mathrm{Ln} 2$ sites is low (ca. 10\%), ${ }^{5}$ the structure is closer to that of a $1 \mathrm{D}$ system, rather than a $2 \mathrm{D}$ system. The unusual dimensionality of the Ln-AV-20 system clearly exerts a considerable influence on the energy transfer dynamics and any kinetic model we employ must therefore also account for this.

3.5. ProgClusters: A Computer Program for Modeling Energy Transfer Dynamics in Systems of Unusual Dimensionality. The energy transfer dynamics between $\mathrm{Ln}^{3+}$ donoracceptor pairs in Ln-AV-20 systems was modeled on a PC using ProgClusters as follows.

Construction of the Model Structure for a Ln-AV-20 Layer. We consider a large 2D array of atomic sites made up of parallel chains of Ln1 sites separated from each other by adjacent chains of potential Ln2 sites. Each Ln1 site will be occupied with a lanthanide ion, but Ln2 sites are randomly occupied or left empty with a probability given by the fraction of Ln2 sites required, i.e., in this work $5 \%$ of potential $\mathrm{Ln} 2$ sites are filled with a Ln ion (see later). The final step in creation of the model structure is to fill each of these occupied Ln1 and Ln2 sites with the specific donor-acceptor lanthanide ions of interest, randomly, but with a probability given by the ratio of donor and acceptor ions in the sample.

Excitation, Energy Transfer and Array Size. All donor ions have equal probability of excitation. After excitation, they will transfer energy to their neighboring ions. In systems where each ion has the same number of identical neighbors, it is possible to model energy migration by a series of steps, or energy transfer hops, in which all of the energy in an excited site is shared equally between neighbors, ${ }^{27,28}$ and a series of such steps very quickly reproduces the energy migration and energy distribution for an infinite number of such sites. The situation is more complicated in the Ln-AV-20 structure, because the number of neighbors can vary between two and four. In this situation, depletion of energy from excited sites with four neighbors will be twice as fast as those with two neighbors. Because of this we limit the amount of energy transferred from an excited site to a neighbor to only $2.5 \%$ of the energy in the excited-site. The choice of how much energy to transfer is a compromise between how accurately the energy transfer is to be modeled over a few energy transfer steps, i.e., short times, and how much computing time is available for the program. For short times, the less energy transferred, the more accurate the simulation, but over even a moderate number of energy transfer steps, simulations using $2.5 \%$ and $25 \%$ transfer are identical. The advantage of having the possibility for a variable amount of energy transferred is that different energy transfer efficiencies into different adjacent ions can be introduced into the program, although in this work we kept the rates for $\mathrm{D} \rightarrow \mathrm{D}$ and $\mathrm{D} \rightarrow \mathrm{A}$ energy transfer the same and made the $\mathrm{A} \rightarrow \mathrm{D}$ rate zero, i.e., $\mathrm{D}$ $\rightarrow$ A energy transfer is irreversible and any energy transferred into an acceptor is lost from the array.

The size of the array used is again a compromise between making it large enough to be truly representative of the random distribution of $\operatorname{Ln} 2$ site and donor-acceptor ions and the computing time available. There is a further complication and that is the effect of energy transfer right to the edge of the array. We found that an array of $900 \times 900$ was large enough to give reproducible energy transfer kinetics, and provided that only those acceptor sites in the middle ninth of the array were initially excited, the amount of energy to reach the edge of the array was negligible, even after the 10000000 energy steps used in the simulation (as can be shown by the binominal distribution). Since we can treat each excitation event and the resultant energy transfer steps independently, the program runs by exciting every donor $\mathrm{Ln}^{3+}$ ion in the central ninth of the array and following the energy transfer steps of arising from all initial excited donors simultaneously.

For each sample, the excited donor decay was modeled using three ProgClusters simulations, which gave an indication of the run-to-run variation arising from the random nature of the distribution of $\mathrm{Ln} 2$ sites and donor-acceptor ions and, therefore, the error associated with the fits.

The amount of energy in excited donor sites as a function of the number of energy transfer steps, which is the raw data obtained at the end of the program simulation, is transformed into a decay curve simulation using a Microsoft 2000 Excel spreadsheet by introducing the following fitting parameters: (i) $I_{\mathrm{F}}$, the fraction of initial excited donor states remaining after the instrument "dead-time", which is used to normalize the experimental decay curves at $t=0$; (ii) $t_{\mathrm{h}}$, the step time, which is a fitting parameter that converts the decay curves described in terms of the "number of steps" generated by the program into "real" time to allow comparison with the experimental decay curves by $t(\mathrm{~s})=$ number of steps $\times t_{\mathrm{h}}(\mathrm{s})$; and (iii) $k_{\text {nat }}$, the natural donor decay rate, which includes all nonradiative and radiative decay processes excluding energy transfer. The 
natural decay is incorporated into the ProgClusters data to give the final curve fit using

$$
I_{t}=F_{t} \exp \left(-k_{\text {nat }} t_{\mathrm{h}}\right)
$$

where $I_{t}$ is the emission intensity and $F_{t}$ is the fraction of excited donors present (calculated by ProgClusters) at time, $t$.

Preliminary curve fits were performed on the $\mathrm{Tb}^{3+}$ emission decay curve for the $\mathrm{Tb}_{1-x} \mathrm{Eu}_{x}-\mathrm{AV}-20$ system, where the fraction of $\mathrm{Eu}^{3+}$ acceptors, $x$, is 0.01. Several physical and fitting parameters were considered during the optimization of the fitting process. As expected, changing the number of $\mathrm{Ln} 2$ sites resulted in considerable variation in the shape of the curve fits. The best curve fits were obtained using $5 \% \mathrm{Ln} 2$ sites, which is in reasonable agreement with the ca. $10 \%$ Ln 2 shown by powder XRD for an Eu-AV-20 sample studied previously. ${ }^{5}$ ProgClusters enables the user to vary the relative rates of $\mathrm{D} \rightarrow \mathrm{D}, \mathrm{D} \rightarrow \mathrm{A}$, Ln1 $\leftrightarrow$ Ln1, and Ln1 $\leftrightarrow$ Ln2 energy transfer. The interatomic distances for $\mathrm{Ln} 1-\mathrm{Ln} 1$ and $\mathrm{Ln} 1-\mathrm{Ln} 2$ sites within an individual layer are 3.951 and $3.626 \AA$, respectively, in Eu-AV-20.5 On the basis of a dipole-dipole interaction $\left(R^{-6}\right)$, it seems reasonable that the relative rate of $\operatorname{Ln} 1 \leftrightarrow \operatorname{Ln} 2$ energy transfer should be ca. 1.7 times greater than the Ln1 $\leftrightarrow$ Ln1 energy transfer rate. However, in the absence of rigorous statistical analysis, it is impossible to determine if the introduction of such a small difference in relative rates into the fitting procedure is meaningful. Thus, to avoid overparametrizing, the relative rates of $\operatorname{Ln} 1 \leftrightarrow \operatorname{Ln} 1$ and $\operatorname{Ln} 1 \leftrightarrow \operatorname{Ln} 2$ and also D $\rightarrow$ D and D $\rightarrow$ A energy transfer were kept the same.

The best curve fits were obtained using $k_{\text {nat }}=50 \mathrm{~s}^{-1}$, particularly toward the tail of the donor decay curve where radiative decay processes are expected to dominate. In the absence of energy transfer and concentration quenching, $\mathrm{Tb}^{3+}$ would be expected to exhibit a long natural lifetime. Tb-AV20 has an emission quantum yield, $\Phi_{\mathrm{em}}$, of $17 \%$ and the observed lifetime, $\tau_{\mathrm{obs}}$, of this sample was ca. $3 \mathrm{~ms} .{ }^{21}$ If radiative decay is $100 \%$ efficient (i.e., $\Phi_{\mathrm{em}}=100 \%$ ), this would correspond to a radiative lifetime $\tau_{\text {rad }} \approx 18 \mathrm{~ms}$ and a subsequent $k_{\text {nat }} \approx 56 \mathrm{~s}^{-1}$. On the basis of these calculations, $k_{\text {nat }}=50 \mathrm{~s}^{-1}$ seems a sensible fitting parameter. The step times required to give reasonable curve fits to the experimental decay curve are on the order of $10^{-9} \mathrm{~s}$.

On the basis of this optimization process, curve fits were performed on the $\mathrm{Tb}^{3+}$ emission decay curves for the entire $\mathrm{Tb}_{1-x} \mathrm{Eu}_{x}$-AV-20 series. Three repeat program runs were carried out for each sample, with a new array set up for each run. The fraction of Ln2 sites was fixed at 5\%, the number of acceptor sites was taken from the EDS measurements, and $k_{\text {nat }}$ was fixed at $50 \mathrm{~s}^{-1}$. $I_{\mathrm{F}}$ was optimized for each individual sample and the step time was allowed to vary freely for each sample and each sample run, allowing a mean value to be calculated.

The same process was also applied to fit the $\mathrm{Gd}^{3+}$ curves of the $\mathrm{Gd}_{1-x} \mathrm{~Tb}_{x}-\mathrm{AV}-20$ series. Here the best fits were obtained with $k_{\text {nat }}=800 \mathrm{~s}^{-1}$, which is in agreement with the much faster emission decay curves observed for $\mathrm{Gd}^{3+}$ compared to $\mathrm{Tb}^{3+}$ (Figure 4). Figures 7 and 8 shows the optimized curve fits for the $\mathrm{Tb}_{1-x} \mathrm{Eu}_{x}-\mathrm{AV}-20$ and $\mathrm{Gd}_{1-x} \mathrm{~Tb}_{x}-\mathrm{AV}-20$ series, respectively, and the fitting data is summarized in Table 1.

As the acceptor concentration increases, a corresponding decrease in the fraction of initial excited donor states remaining after the instrument dead-time, $I_{\mathrm{F}}$, is observed in both systems. With increasing acceptor concentration, the donor emission lifetime decreases due to $\mathrm{D} \rightarrow$ A energy transfer, and consequently, due to the time-resolution constraints of the instrument, the total amount of the decay curve that is measured also

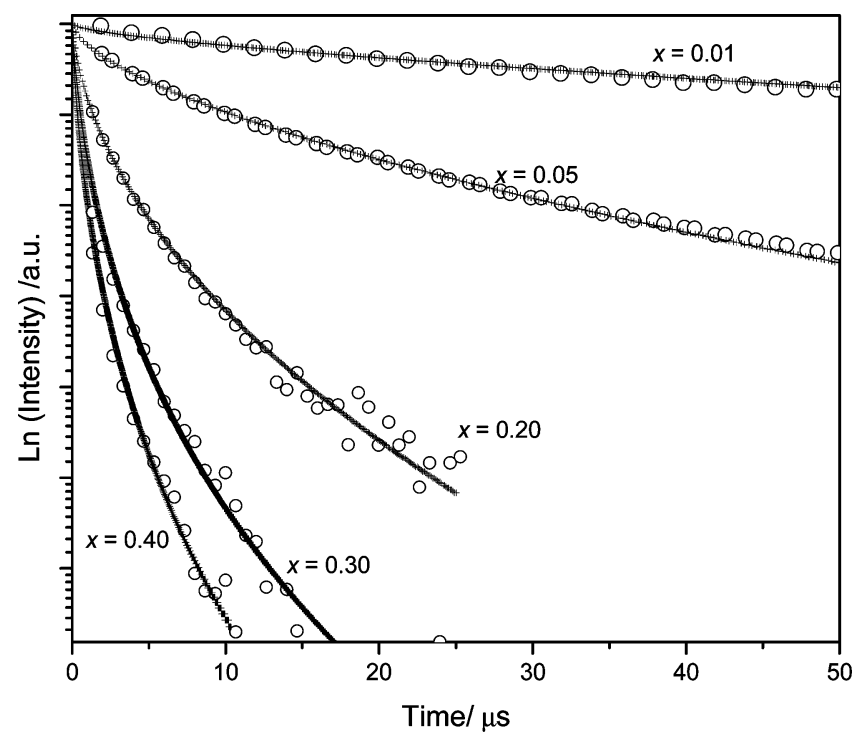

Figure 7. Optimized curve fits (crosses) obtained from ProgClusters for the experimental $300 \mathrm{~K} \mathrm{~Tb}^{3+}\left({ }^{5} \mathrm{D}_{4}\right)$ decays $\left(\lambda_{\mathrm{ex}}=355 \mathrm{~nm}, \lambda_{\mathrm{em}}=\right.$ $542 \mathrm{~nm}$, open circles) of the $\mathrm{Tb}_{1-x} \mathrm{Eu}_{x}-\mathrm{AV}-20$ series. Curve fits performed as described in the text.

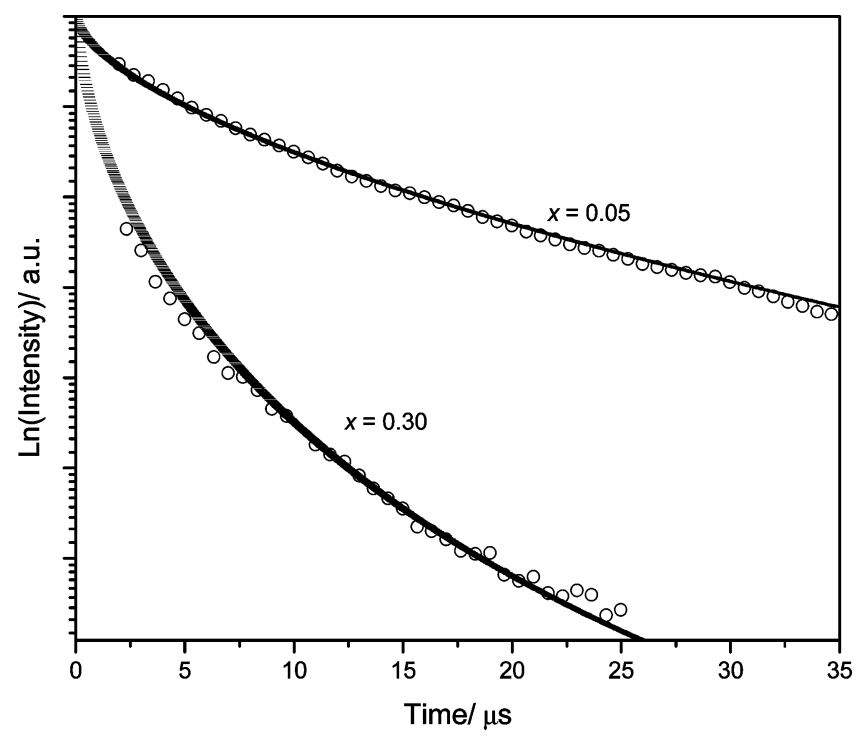

Figure 8. Optimized curve fits (dashed lines) obtained from ProgClusters for the experimental $300 \mathrm{~K} \mathrm{Gd}^{3+}\left({ }^{6} \mathrm{P}_{7 / 2}\right)$ decays $\left(\lambda_{\mathrm{ex}}=266\right.$ $\mathrm{nm}, \lambda_{\mathrm{em}}=313 \mathrm{~nm}$ ) of the $\mathrm{Gd}_{1-x} \mathrm{~Tb}_{x}-\mathrm{AV}-20$ series. Curve fits performed as described in the text.

decreases. In effect, $I_{\mathrm{F}}$ corresponds to the fraction of the true decay curve that is actually measured, and at the highest acceptor concentrations this is only the last $10 \%$ (Table 1). The step times $\left(t_{\mathrm{h}} \sim 4.81 \times 10^{-9} \mathrm{~s}\right)$ are the same within experimental error for the entire $\mathrm{Tb}_{1-x} \mathrm{Eu}_{x}-\mathrm{AV}-20$ series, with the exception of the $x$ $=0.01$ sample $\left[t_{\mathrm{h}}=(3.71 \pm 0.21) \times 10^{-9} \mathrm{~s}\right]$, which is slightly slower.

The rate constant for energy transfer between nearest neighbor ions, $k_{\mathrm{NN}}$, may be determined after $n$ energy steps from

$$
I_{t}=I_{0} \exp \left(-k_{\mathrm{NN}} t_{\mathrm{h}}\right)
$$

where $I_{0}$ is the normalized emission intensity at $t=0$ and $t_{\mathrm{h}}$ is the step time. Since the amount of energy transferred per hop is fixed in ProgClusters as 0.025, then after one hop, $I_{\mathrm{t}}=$ $1-0.025$, i.e. 


$$
\ln \left(\frac{0.975}{1}\right)=k_{\mathrm{NN}} t_{\mathrm{h}}
$$

The calculated energy transfer rate constants are shown in Table 1 . With the exception of the $x=0.01$ sample, $k_{\mathrm{NN}}$ is the same within experimental error for the entire $\mathrm{Tb}_{1-x} \mathrm{Eu}_{x}-\mathrm{AV}-20$ series, with a mean value of $k_{\mathrm{NN}}=(5.30 \pm 0.07) \times 10^{6} \mathrm{~s}^{-1}$. In the $x$ $=0.01$ sample, the fraction of Ln2 sites is expected to be particularly important. The 5\% Ln2 sites used as a fitting parameter here may therefore not be optimum for this sample, which may go some way to explaining the small differences observed. A similar trend is observed for the $\mathrm{Gd}_{1-x} \mathrm{~Tb}_{x}-\mathrm{AV}-20$ series, with the mean $k_{\mathrm{NN}}=(5.96 \pm 0.13) \times 10^{6} \mathrm{~s}^{-1}$. The slightly faster rate constant observed for the $\mathrm{Gd}_{1-x} \mathrm{~Tb}_{x}-\mathrm{AV}-20$ series is consistent with the fast $\mathrm{Gd}^{3+} \rightarrow \mathrm{Gd}^{3+}$ energy migration rates reported by other workers. ${ }^{29}$

The number of energy hops, $n_{\text {hop }}$, required to arrive at a trap site in our dynamic energy transfer regime is estimated for each acceptor concentration in the $\mathrm{Tb}_{1-x} \mathrm{Eu}_{x}-\mathrm{AV}-20$ series from

$$
n_{\text {hop }}=k_{\mathrm{NN}} / k_{\mathrm{DA}}
$$

As expected at very low acceptor concentrations, on average the energy must migrate fairly large distances ( $>1000$ steps) over the donor system before arriving at an energy acceptor (Table 1). With increasing $\mathrm{Eu}^{3+}$ concentration the distance between nearest-neighbor $\mathrm{D}-\mathrm{A}$ pairs decreases rapidly, and consequently, the energy migration within the donor ion system becomes much less important $(\sim 15$ steps for $x=0.40)$.

3.6. Tuning the Dimensionality of the Ln-AV-20 Layers. Since, at high acceptor concentrations, energy migration and transfer in the Ln1 linear chains become more important, at this limit the energy transfer dynamics should tend toward a 1D trapping model. Random walks in 1D systems with annihilation by trapping have been intensively studied in the last 2 decades. ${ }^{28,30-36}$ The approach we use here is the decoupled-ring approximation. ${ }^{36} \mathrm{We}$ consider a linear onedimensional chain of infinite regular lattice sites, in which a fraction, $f$, of the lattice sites are replaced by traps. A trap is simply a site where an excited-state may undergo spontaneous decay with a fixed probability $0<q \leq 1$, which may be used to represent an acceptor ion in mixed Ln-AV-20 systems. Following the excitation pulse, an initial distribution of excited states will be formed randomly over the lattice sites. It is assumed that each excited state may undergo a symmetric random walk, such that an excited state initially located at site $k$ at time $t$ can subsequently be found at either $\left(k-1, t+t_{\mathrm{h}}\right)$ or $\left(k+1, t+t_{\mathrm{h}}\right)$ both with probability $P=0.5$, where $t_{\mathrm{h}}$ represents the physical time-step or hop. At each hop, every migrating excited state may lose energy through two mutually exclusive mechanisms: (i) spontaneous radiative decay with fixed probability $0 \leq p<1$ and (ii) nonradiative quenching by a trap site with a given probability $0<q \leq 1$. Using the decoupled-ring approximation, the linear 1D chains are decoupled by cutting the infinite line at each trap and bending each segment onto itself to form a ring (Figure 9). ${ }^{36}$ The entire chain is thereby mapped onto an infinite set of rings of varying size, $n$. For a random initial distribution of traps with concentration $0<a<1$, all values of $n$ are possible. The probability of finding a ring of size $n$ is the geometric function. Thus, the probability of site $N$ not being a trap site is given by $a(1-$ $a)^{n-1}$, where $n$ is the ring size, i.e., the total number of lattice

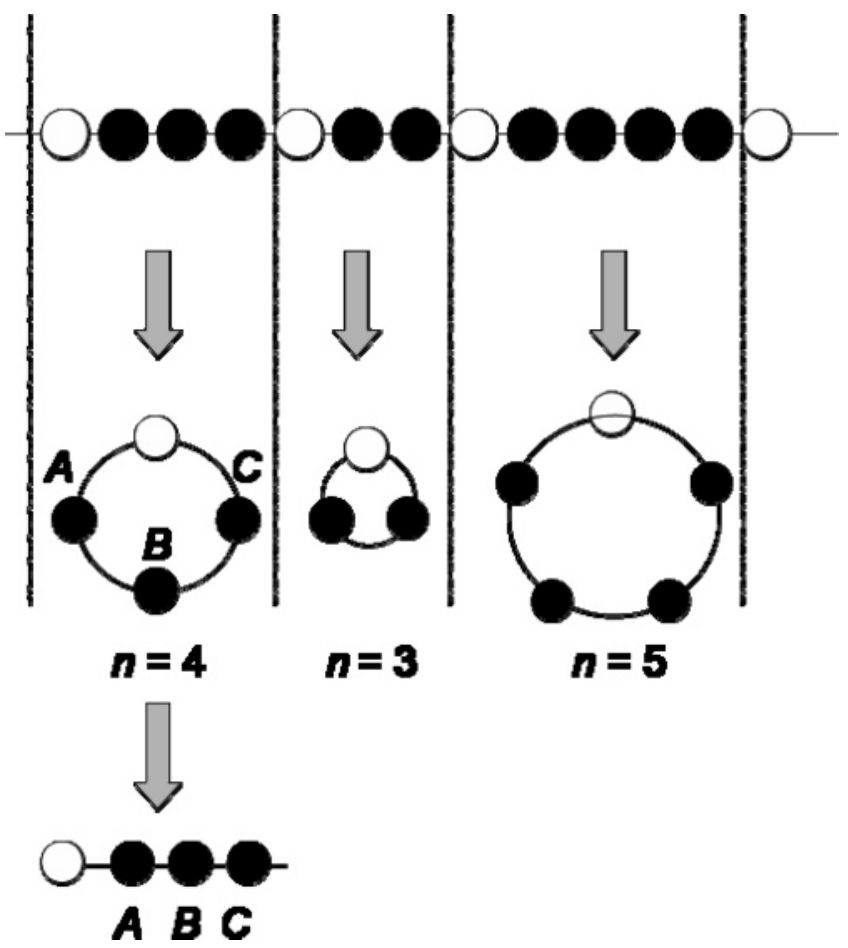

Figure 9. Schematic representation of the ring-coupling procedure adapted from ref 36 . The black circles represent regular lattice sites and the white circles represent trap sites. The infinite one-dimensional chain is divided into segments determined by the location of the trap sites, and each segment is then folded back on itself to form a ring.

and trap sites in that segment. The probability of finding a ring of size $n$ is given by ${ }^{36}$

$$
P(n)=n a^{2}(1-a)^{n-1}
$$

Given an arbitrary distribution of traps, it is possible to obtain the full-chain solution for the donor ion survival probability. A linear chain of lattice sites of length $l=(n-1)$ is set up. One trap site is placed at either end of the chain such that the total chain length $=(n+1)$. At $t=0$, all lattice sites are excited, after this time the excitation energy undergoes a symmetrical random walk. Energy transfer into a trap results in annihilation of the energy packet. If the decay curves for each individual ring size are normalized and correctly weighted with the relative abundance for the occurrence of rings of length $n$ in the chain from eq 8 , the summation of the final weighted decay curves will give the total decay function for all chains. Figure 10 shows the best fits obtained for the $\mathrm{Tb}^{3+}$ emission decay curve of the $\mathrm{Tb}_{60} \mathrm{Eu}_{40}-\mathrm{AV}-20$ sample using the decoupled ring approximation and ProgClusters. The fits obtained by both models are in good agreement with the experimental decay curve. For the 1D random walk, the best fit is obtained with $t_{\mathrm{h}}=1.68 \times 10^{-7} \mathrm{~s}$, while for the ProgClusters $t_{\mathrm{h}}=(4.80 \pm 0.12) \times 10^{-9} \mathrm{~s}$ gives the best fit. However, in the 1D random walk model, the probability that energy is transferred to each nearest neighbor is $P=0.5$, while $P=0.025$ for ProgClusters. Taking this into account, the corresponding hop time for the ProgClusters, where $P=0.5$, is $t_{\mathrm{h}}=1.0 \times 10^{-7} \mathrm{~s}$, which is in good agreement with that of the $1 \mathrm{D}$ random walk model. The difference in $t_{\mathrm{h}}$ obtained by the two approaches is due to the inclusion of 5\% Ln2 sites in the ProgClusters model, which shifts the system dimensionality slightly away from a purely $1 \mathrm{D}$ regime.

Thus, by varying the relative donor and acceptor concentrations, it is possible to tune the dimensionality of the Ln-AV-20 between 1D and 2D. At low acceptor concentrations, the 


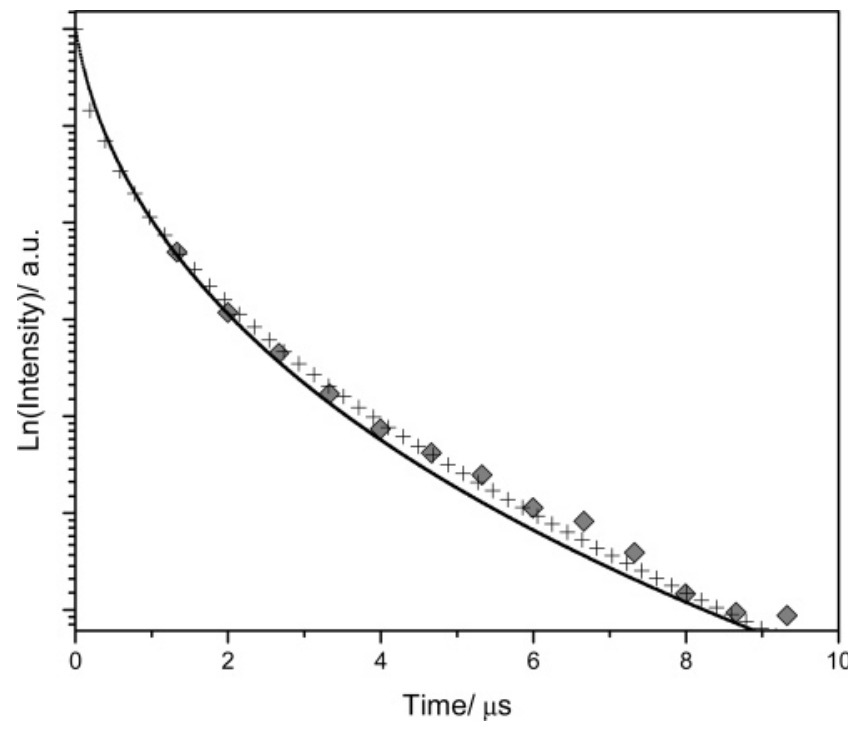

Figure 10. Optimized curve fits to the $\mathrm{Tb}^{3+}\left({ }^{5} \mathrm{D}_{4}\right)$ decay curve (filled diamonds) for $\mathrm{Tb}_{1-x} \mathrm{Eu}_{x}-\mathrm{AV}-20(x=0.4)$ obtained by (i) the decoupled ring approximation (1D, crosses) and (ii) ProgClusters (5\% Ln2 sites, solid line; see Table 1 for fitting parameters). Curve fits as described in the text.

excitation energy has to migrate fairly large distances over the donor ion system before arriving at the acceptor site. In this limit the presence of the Ln2 bridging sites is very important in controlling the energy transfer dynamics. However, as the acceptor ion concentration increases, most donor ions are effectively situated just a few hops away from an acceptor site, and consequently, energy transfer in the linear Ln1 chains dominates the decay dynamics which therefore approach the $1 \mathrm{D}$ regime.

\section{Conclusions}

We have shown that the presence of two $\mathrm{Ln}^{3+}$ environments results in an unusual dimensionality of the Ln-AV-20 layers and complex $\mathrm{D} \rightarrow$ A energy transfer dynamics that cannot be modeled using conventional energy transfer models. This problem has been overcome by development of a computer modeling program designed to model energy transfer between nearest-neighbor $\mathrm{Ln}^{3+}$ pairs within the AV-20 layers. With the aid of this program, it has been possible to fully model and interpret the complex energy transfer processes that occur in mixed Ln-AV-20 systems. At high acceptor concentrations, it has been shown that energy transfer in short donor chains dominates and kinetics tends toward one-dimensional behavior. Therefore, in mixed Ln-AV-20 systems, it is possible to tune the dimensionality of the AV-20 layers between the $1 \mathrm{D}$ and intermediate $1 \mathrm{D}-2 \mathrm{D}$ regimes by controlling the relative donor and acceptor concentrations.

Although ProgClusters was designed with the AV-20 system in mind, it is not specific to this structure and in principle may be applied to any system whose dimensionality ranges between 1D and 2D. ProgClusters offers the introduction of several other modeling parameters, such as relative transfer rates and transfer between layers, as well as providing information about the decay kinetics of specific donor sites, none of which were required for studying the AV-20 system. Moreover, it is possible to envisage further developments to this program, which, for example, enable information about emission colors for specific $\mathrm{Ln}^{3+}$ combinations to be introduced, thus presenting the possibility of modeling the emission color of a material as a function of the individual $\mathrm{Ln}^{3+}$ emission decay components over time. We believe that the possibility of determining the required relative $\mathrm{Ln}^{3+}$ concentrations in this way to design a material with a specific emission color prior to experimentation is an extremely useful tool in the development of these, and related materials, for display technologies.

Acknowledgment. R.C.E. thanks the European Union Marie Curie Training Site (MCFH-1999-00663) for provision of a fellowship (HPMT-GH-00-00206-12) to fund her stay at the University of Aveiro and the University of Wales Swansea for provision of a research studentship.

\section{References and Notes}

(1) Blasse, G.; Grabmeier, B. C. Luminescent Materials; Springer: Berlin, 1994.

(2) Jüstel, T.; Nikol, H.; Ronda, C. R. Angew. Chem., Int. Ed. 1998 37, 3084.

(3) Rocha, J.; Anderson, M. W. Eur. J. Inorg. Chem. 2000, 801.

(4) Ananias, D.; Ferreira, A.; Rocha, J.; Ferreira, P.; Rainho, J. P.; Morais, C. M.; Carlos, L. D. J. Am. Chem. Soc. 2001, 123, 5735.

(5) Ferreira, A.; Ananias, D.; Carlos, L. D.; Morais, C. M.; Rocha, J. J. Am. Chem. Soc. 2003, 125, 14573.

(6) Ananias, D.; Kostova, M.; Paz, F. A. A.; Ferreira, A.; Carlos, L. D.; Klinowski, J.; Rocha, J. J. Am. Chem. Soc. 2004, 126, 10410.

(7) Kostova, M. H.; Sá Ferreira, R. A.; Ananias, D.; Carlos, L. D.; Rocha, J. J. Phys. Chem. B 2006, 110, 15312.

(8) Kostova, M. H.; Ananias, D.; Paz, F. A. A.; Ferreira, A.; Rocha, J.; Carlos, L. D. J. Phys. Chem. B 2007, 111, 3576. 268.

(9) Ouyang, X.; Kitai, A. H.; Siegele, R. Thin Solid Films 1995, 254,

(10) Banerjee, A. K.; Stewart-Darling, F.; Flint, C. D.; Schwartz, R. W. J. Phys. Chem. 1981, 85, 146.

(11) Bettinelli, M.; Flint, C. D. J. Phys.: Condens. Matter 1990, 2, 8417.

(12) Luxbacher, T.; Fritzer, H. P.; Flint, C. D. Spectrochim. Acta A 1998, $54,2027$.

(13) Moadhen, A.; Elhouichet, H.; Canut, B.; Sandu, C. S.; Ouestlati, M.; Roger, J. A. Mater. Sci. Eng. 2003, B105, 157.

(14) Takeuchi, N.; Ishida, S.; Matsumura, A.; Ishikawa, Y.-I. J. Phys. Chem. B 2004, 108, 12397.

(15) ProgClusters was developed by Alastair Douglas and can be found at www.waxpad.com/articles/clusters.html.

(16) Albin, M.; Horrocks, W. D. Inorg. Chem. 1985, 24, 895.

(17) Weber, M. J. Phys. Rev. B 1971, 4, 2932.

(18) Kudo, A.; Sakata, T. J. Phys. Chem. 1995, 99, 15963.

(19) Honma, T.; Toda, K.; Ye, Z.-G.; Sato, M. J. Phys. Chem. Solids 1998, 59, 1187.

(20) Toda, K.; Honma, T.; Sato, M. J. Lumin. 1997, 71, 71.

(21) Evans, R. C. Ph.D. Thesis, University of Wales Swansea, 2007.

(22) Yokota, M.; Tanimoto, O. J. Phys. Soc. Jpn. 1967, 22, 779.

(23) Lakowicz, J. R. Principles of Fluorescence Spectroscopy; Springer: Berlin, 2006.

(24) Kubota, S.-I.; Shimada, M.; Takizawa, H.; Endo, T. J. Alloys Compd. 1996, 241, 16.

(25) Berdowski, P. A. M.; Herk, J. V.; Blasse, G. J. Lumin. 1985, 34,

(26) Huber, D. L. Phys. Rev. 1979, 20, 2307.

(27) Yuste, S. B.; Acedo, L. Physica A 2003, 336, 334.

(28) Klafter, J.; Silbey, R. J. Chem. Phys. 1980, 72, 849.

(29) van Schaik, W.; van Heek, M. M. E.; Middel, W.; Blasse, G. J Lumin. 1995, 63, 103.

(30) Donsker, M. D.; Varadhan, S. R. S. Commun. Pure Appl. Math. 1979, 32, 721 .

(31) Havlin, S.; Weiss, G. H.; Kiefer, J. E.; Dishon, M. J. Phys. A: Math. Gen. 1984, 17, L347.

(32) Anlauf, J. K. Phys. Rev. Lett. 1984, 52, 1845.

(33) Weiss, G. H.; Havlin, S. J. Stat. Phys. 1984, 37, 17.

(34) Weiss, G. H.; Havlin, S. J. Chem. Phys. 1985, 83, 5670.

(35) Weiss, G. H. Aspects and Applications of the Random Walk; NorthHolland: Amsterdam, 1994.

(36) Aspelmeier, T.; Magnin, J.; Graupner, W.; Täubner, U. C. Physica A 2006. 